\title{
Caveolin-1 as a potential high-risk prostate cancer biomarker
}

\author{
JAROMIR GUMULEC $^{1,2}$, JIRI SOCHOR ${ }^{2,3}$, MARIAN HLAVNA ${ }^{1}$, MARKETA SZTALMACHOVA ${ }^{1,2}$, \\ SONA KRIZKOVA ${ }^{2,3}$, PETR BABULA ${ }^{2,3}$, ROMAN HRABEC ${ }^{4}$, ARNE ROVNY ${ }^{4}$, VOJTECH ADAM ${ }^{2,3}$, \\ TOMAS ECKSCHLAGER ${ }^{5}$, RENE KIZEK ${ }^{2,3}$ and MICHAL MASARIK ${ }^{1}$ \\ ${ }^{1}$ Department of Pathological Physiology, Faculty of Medicine, Masaryk University, CZ-625 00 Brno; ${ }^{2}$ Department of \\ Chemistry and Biochemistry, Mendel University in Brno, CZ-613 00 Brno; ${ }^{3}$ Central European Institute of \\ Technology, Brno University of Technology, CZ-616 00 Brno; ${ }^{4}$ Department of Urology, St. Anne's \\ University Hospital Brno, CZ-656 91 Brno; ${ }^{5}$ Department of Paediatric Haematology and Oncology, \\ 2nd Faculty of Medicine Charles University, CZ-150 06 Prague 5, Czech Republic
}

Received October 4, 2011; Accepted November 7, 2011

DOI: $10.3892 /$ or.2011.1587

\begin{abstract}
Current diagnostic techniques of prostate cancer cannot efficiently distinguish the latent and low-risk forms from the high-risk significant forms of prostate cancer. Caveolin-1 (Cav-1), except other functions, plays an important role in cell transformation and the process of tumorigenesis. Furthermore, Cav-1 is involved in metastatic processes. It has also been shown that Cav-1 expression is induced under stress conditions, such as oxidative stress. The present study focused on the determination of prognostic markers of aggressive (high-grade) forms of prostate cancer. We determined serum Cav-1 and serum markers of antioxidant activity-glutathione (GSH), 2,2-diphenyl-1-picrylhydrazyl (DPPH), Trolox equivalent antioxidant capacity (TEAC), ferric-reducing antioxidant power (FRAP), N,N-dimethyl-1,4-diaminobenzene (DMPD), free radicals method (FRK) and blue chromium peroxide (Cro) in 97 serum samples (82 prostate cancer patients and 15 controls). We found insignificant differences in Cav-1 between the sera of patients and controls (5.69 in the cancer group vs. $5.42 \mathrm{ng} / \mathrm{ml}$ in the control group). However, we found a significant $(\mathrm{p}<0.004) 2.8$-fold elevation of Cav-1 in high tumour stages (TNM T4) compared to lower stages and a significant positive association with histological grading $(r=0.29, p=0.028)$. We also found that in patients with high serum Cav-1 the antioxidant capacity of the body is reduced. These findings indicate that Cav-1 may be an interesting tool for the prediction of disease burden.
\end{abstract}

Correspondence to: Dr Michal Masarik, Department of Pathological Physiology, Faculty of Medicine, Masaryk University, Kamenice 5, CZ-625 00 Brno, Czech Republic

E-mail: masarik@med.muni.cz

Key words: prostate cancer, tumour marker, prognostic marker, immunodetection, electrochemistry, polymerase chain reaction, molecular biology techniques

\section{Introduction}

Discovering and definition of new biochemical markers, which are specifically connected with grave pathological states including tumour diseases, are among the most important objectives of biomedical research. Identification of highly specific and sensitive biomarkers represents the main aim of modern research, because only such biomarkers may be applied towards the early diagnosis of malignant disease, prediction of prognosis and eventually development of an appropriate treatment strategy in clinical practice (1). Malignant tumours occupy the first position among diagnosed diseases due to the improvement of health care. The process of genesis of a tumour cell includes accumulation of alterations in a cell genome, which may develop for decades. Mechanisms of the cell cycle and apoptosis regulation play a crucial role in the protection against these changes.

Prostate carcinoma is the most frequent malignant disease among men in the Czech Republic. Global data about the incidence of prostate carcinoma are not too exact, especially due to fact that a lot of men die due to this disease without its clinical manifestations (2). Substantial progress in the diagnosis of tumour diseases has been observed along with the development of proteomics due to the identification of new tumour markers (3-8). These markers, usually proteins, are closely connected with the development and eventually the progression of the disease and are present in tumour cells in altered concentrations.

Due to the introduction of prostate-specific antigen (PSA) screening in prostate cancer diagnosis, the incidence of this disease has increased by $>50 \%$ in the recent years. At present, its incidence is higher in comparison with bronchogenic carcinoma with almost half of the men at the age of 80 suffering from prostate carcinoma. PSA represents the routinely used diagnostic marker of prostate carcinoma (9-11). Measurements of PSA blood serum levels began in the early 1980s. There are plenty of methods used for determination of PSA blood serum levels $(10,12)$. However, determination of PSA is not specific and sensitive in the blood serum and its positive predictive value is only about $35 \%$. PSA may also be elevated in various 
non-tumour conditions in healthy men. Its level is increased in other prostate diseases (benign prostatic hyperplasia, prostatitis and prostate infections) or with physical activity including cycling. Almost two thirds of men with increased PSA are healthy. Contrariwise, $20 \%$ of men suffering from prostate carcinoma have normal PSA levels.

Therefore, it is not surprising that searching for new markers for this type of tumour disease is still a focus of research. Caveolin is a protein, which is often associated with tumour disease as a potential tumour marker. It is an integral membrane protein and important integral part of caveolae membranes. Its presence was already discovered in caveolae membranes in 1953 (13). These membranes are involved in receptor-independent endocytosis. Caveolae are microdomains of lipid rafts, which are rich in sphingolipids and cholesterol and play an essential role in the degradation of cholesterol (14). However, they also participate in transmembrane signalling. There are three known types of caveolin, which differ in their molecular structure and tissue distribution. Caveolin-1 is profusely present in adipocytes, epithelial cells, pneumocytes and fibroblasts. Caveolin-2 is expressed in the cells of mesenchyma, epithelial cells and neuroglia. Caveolin-3 occurs predominantly in muscle cells $(15,16)$. All types of caveolins are investigated in view of the pathogenesis of some diseases (17-20). The connection between caveolin-1 and tumorigenesis has been investigated in many studies (21-24). Caveolin-1 has been demonstrated to regulate cell proliferation, so, it can play an important role in cell transformation and the process of tumorigenesis $(23,25,26)$. Caveolin-1 is also involved in metastatic processes (25). The reason for the participation of caveolin-1 in these processes is the fact that normal epithelial cells are characterized by cell adhesion and the cell is closely connected through the membrane with its surroundings and is able to respond to changes in cell surroundings. Caveolin-1 directly participates in these cell processes, especially due to its connection with integrins. Changes in protein function lead to the lapse of cell functions (adhesion) and cell mobility and the development of metastatic processes (22). Furthermore, it has been demonstrated that caveolin-1 expression is induced under stress conditions, such as oxidative stress $(27,28)$.

Metallothioneins (MT) as a metal-binding proteins represent other promising tumour markers, which are intensely studied in connection with prostate carcinoma among other diseases (29-31). It has been recently demonstrated that metallothioneins play an important role in the development and progression of some tumour diseases (32-42). Enhanced levels of MT in tumour cells are probably closely connected with cell proliferation $(43,44)$. Recent studies point at the overexpression of MT in relation to the metal-based cytostatic agents $(45,46)$. Other potential tumour markers include $\alpha$-methylacyl-CoA-racemase (AMACR). This substance is a peroxisomal and mitochondrial enzyme involved in $\beta$-oxidation of branched fatty acids and in catabolism of bile acids metabolites (47). Increased levels of these proteins have been described in adenocarcinomas and high grade prostatic intraepithelial neoplasia (48). On the other hand, only low levels of this marker are described in benign hyperplasia and in atypical adenomatous hyperplasia $(47,49,50)$.

It is well evidenced that the total antioxidant capacity of the human body is reduced, when patients suffer from a serious disease such as cancer, particularly when the disease is long-term and the patients are of higher age (51-53). Thus, we aimed to determine most of the common markers of antioxidant capacity and to put them into the context with caveolin-1. The originality of this study consists in the analysis and mutual correlation between the above-mentioned tumour markers and the markers of oxidative stress in blood sera of patients suffering from prostate carcinoma. Our results show the necessity for the determination of more tumour markers to aid in the knowledge of the disease stage of the patient and for the identification of optimal treatments.

\section{Materials and methods}

Chemical and biochemical reagents. All chemicals of ACS purity were purchased from Sigma-Aldrich (St. Louis, Mo, USA) unless otherwise noted. The primary mouse metallothionein antibody and the secondary anti-mouse horseradish peroxidase (HRP)-conjugated antibody were purchased from Abcam (Cambridge, MA, USA). The primary rabbit PSA and caveolin-1 antibody and the secondary anti-rabbit HRP-conjugated antibody were purchased from Santa Cruz Biotechnology, Inc. (Santa Cruz, CA, USA). The primary rabbit AMACR antibody was purchased from Clonestar (Czech Republic). For chemiluminiscent detection of Western blot membranes the ECL Western blot detection reagents system from Bio-Rad Laboratories (USA) was used.

Sample preparation for electrochemical analysis. The samples of blood serum were denatured at $99^{\circ} \mathrm{C}$ in a thermomixer (Eppendorf 5430, Germany) for $15 \mathrm{~min}$ with shaking and centrifuged at $15,000 \mathrm{x} \mathrm{g}$ at $4^{\circ} \mathrm{C}$ for $30 \mathrm{~min}$ (Eppendorf 5402). Heat treatment effectively denatures and removes thermolabile and high-molecular-weight proteins from samples. The prepared samples were used for MT and glutathione (GSH) analyses.

Differential pulse voltammetry-Brdicka reaction. Differential pulse voltammetric measurements were performed with the 747 VA Stand instrument connected to the 746 VA Trace Analyzer and the 695 Autosampler (Metrohm, Switzerland), using a standard cell with three electrodes and cooled sample holder $\left(4^{\circ} \mathrm{C}\right)$. A hanging mercury drop electrode (HMDE) with a drop area of $0.4 \mathrm{~mm}^{2}$ was the working electrode. An $\mathrm{Ag} / \mathrm{AgCl} / 3 \mathrm{M} \mathrm{KCl}$ electrode was the reference and glassy carbon electrode was auxiliary. For data processing GPES 4.9 supplied by EcoChemie was employed. The analysed samples were deoxygenated prior to measurements by purging with argon (99.999\%) and saturated with water for $120 \mathrm{sec}$. Brdicka supporting electrolyte containing $1 \mathrm{mM} \mathrm{Co}\left(\mathrm{NH}_{3}\right)_{6} \mathrm{Cl}_{3}$ and $1 \mathrm{M}$ ammonia buffer $\left[\mathrm{NH}_{3}(\mathrm{aq})+\mathrm{NH}_{4} \mathrm{Cl}, \mathrm{pH}\right.$ 9.6] was used. The supporting electrolyte was exchanged after each analysis. The parameters of the measurement were as follows: initial potential of $-0.7 \mathrm{~V}$, end potential of $-1.75 \mathrm{~V}$, modulation time $0.057 \mathrm{sec}$, time interval $0.2 \mathrm{sec}$, step potential $2 \mathrm{mV}$, modulation amplitude $-250 \mathrm{mV}, \mathrm{E}_{\mathrm{ads}}=0 \mathrm{~V}$, volume of injected sample: $20 \mu \mathrm{l}$ (x100 diluted sample with $0.1 \mathrm{M}$ phosphate-buffer $\mathrm{pH}$ 7.0). All experiments were carried out at a temperature of $4^{\circ} \mathrm{C}$ employing the thermostat Julabo F25 (Labortechnik GmbH, Germany). 
Determination of low-molecular-mass thiols. High performance liquid chromatography with an electrochemical detection (HPLC-ED) system consisted of two solvent delivery pumps operating in the range of 0.001-9.999 $\mathrm{ml} / \mathrm{min}$ (Model 582 ESA Inc., Chelmsford, MA), Zorbax Eclipse AAA Column (4.6 x $150 \mathrm{~mm} 3.5-\mu \mathrm{m}$ particle size; Varian Inc., CA, USA), and a CoulArray electrochemical detector (Model 5600A, ESA, USA). The sample (30 $\mu \mathrm{l})$ was injected using an autosampler (Model 540 Microtiter HPLC; ESA, USA). HPLC-ED experimental conditions were as follows. The compositions of the mobile phases were: A, $80 \mathrm{mM}$ trifluoroacetic acid and $\mathrm{B}$, methanol. They were mixed in gradient from $3 \% \mathrm{~B}$ in the first minute, $10 \% \mathrm{~B}$ in the second to the sixth minute and $98 \%$ B from the seventh minute of the separation; flow of the mobile phase was $0.8 \mathrm{ml} / \mathrm{min}$, temperature of the separation was $40^{\circ} \mathrm{C}$; working electrodes potential was $900 \mathrm{mV}$; detector temperature was $30^{\circ} \mathrm{C}$; each measurement was done in triplicates. Retention time of the reduced GSH was 5 min. GSH concentration was calculated from a calibration curve (0.5-100 $\mu \mathrm{M})$. The signal was quantified as a sum of current responses from all working electrodes $(54,55)$. In the case of real sample measurements, the shift of the retention time was of about $\pm 2 \%$.

Determination of antioxidant activity. For determination of antioxidant activity a BS-400 automated spectrophotometer (Mindray, China) was used. It is composed of cuvette space tempered to $37 \pm 1^{\circ} \mathrm{C}$, reagent space with a carousel for reagents (tempered to $4 \pm 1^{\circ} \mathrm{C}$ ), sample space with a carousel for preparation of samples and an optical detector. Transfer of samples and reagents is provided by robotic arm equipped with a dosing needle (error of dosage up to $5 \%$ of volume). Cuvette contents are mixed by an automatic mixer including a stirrer immediately after addition of reagents or samples. Contamination is reduced due to its rinsing system, including rinsing of the dosing needle as well as the stirrer by MilliQ water. For detection itself, the following range of wave lengths can be used - 340, 380, 412, 450, 505, 546, 570, 605, 660, 700, 740 and $800 \mathrm{~nm}$. Experimental details on all used spectrometric assays have been previously described (56).

Sodium dodecyl sulphate polyacrylamide gel electrophoresis (SDS-PAGE). The electrophoresis was performed according to Laemmli using a Mini Protean Tetra apparatus with a gel dimension of $8.3 \times 7.3 \mathrm{~cm}$ (Bio-Rad Laboratories) (57). Firstly we poured $10 \%(\mathrm{~m} / \mathrm{V})$ running gel and then $5 \%(\mathrm{~m} / \mathrm{V})$ stacking gel. The gels were prepared from $30 \%(\mathrm{~m} / \mathrm{V})$ acrylamide stock solution with $1 \%(\mathrm{~m} / \mathrm{V})$ bisacrylamide (SERVA, Germany). The polymerization of the running or stacking gels was carried out at room temperature for $45 \mathrm{~min}$. Prior to analysis the samples were mixed with reducing (DTT, final concentration $400 \mathrm{mM}$ ) sample buffer in 4:1 ratio. The samples were boiled for $5 \mathrm{~min}$ and then the sample was loaded onto the gel. For determination of molecular mass, the protein ladder, broad or lower range (Bio-Rad Laboratories) was used. The electrophoresis ran at $80 \mathrm{~V}$ for 20 min subsequently increased to $120 \mathrm{~V}$ for $1 \mathrm{~h}$ (Power Basic, Bio-Rad Laboratories) in Tris-glycine buffer (0.025 M Trizma-base, $0.19 \mathrm{M}$ glycine and $0.0035 \mathrm{M}$ SDS, pH 8.3). Silver staining of the gels was performed using the Bio-Rad Silver stain kit according to Merril et al (58).
Western blot analysis. After the electrophoretic separation, the proteins were transferred onto a nitrocellulose membrane (Bio-Rad Laboratories) in a Bio-Rad apparatus. The blotting was carried out for $1 \mathrm{~h}$ at a constant current of $0.9 \mathrm{~mA}$ for $1 \mathrm{~cm}^{2}$ of the membrane. After the transfer, the membrane was blocked in 5\% non-fat milk in PBS $(137 \mathrm{mM} \mathrm{NaCl}$, $2.7 \mathrm{mM} \mathrm{KCl}, 1.4 \mathrm{mM} \mathrm{NaH}_{2} \mathrm{PO}_{4}$, and $4.3 \mathrm{mM} \mathrm{Na}_{2} \mathrm{HPO}_{4}$; $\mathrm{pH}$ 7.4) for $2 \mathrm{~h}$. The incubation with mouse primary antibody in dilution of 1:750 in PBS with 5\% non-fat milk was carried out for $12 \mathrm{~h}$ at $4{ }^{\circ} \mathrm{C}$. After three washing with PBS containing $0.05 \%(\mathrm{v} / \mathrm{v})$ Tween-20 (PBS-T) for $5 \mathrm{~min}$ the membrane was incubated with secondary antibody (anti-mouse labelled with HRP, Sigma-Aldrich, diluted 1:5,000) for $1 \mathrm{~h}$ at room temperature. Then, the membrane was washed three times with PBS-T for $5 \mathrm{~min}$ and incubated with the ECL WB detection reagents (Santa Cruz Biotechnology Inc.).

Dot-immunobinding assay. For immunobinding assays PVDF membranes (Bio-Rad Laboratories) were used. The sample $(1 \mu \mathrm{l})$ was applied and dried. Further the membrane was blocked in $2 \%$ bovine serum albumin (BSA) in PBS for $0.5 \mathrm{~h}$ with constant shaking. The incubation with the primary antibody (1:500 diluted) was carried out for $1 \mathrm{~h}$ at $37^{\circ} \mathrm{C}$. After three times repeated washing in PBS containing $0.05 \%$ (v/v) Tween-20 (0.05\% PBS-T) for $5 \mathrm{~min}$, the membrane was incubated in the presence of secondary antibody at a dilution 1:5,000 for $1 \mathrm{~h}$ at $37 \mathrm{C}$. Then the membrane was washed three times in $0.05 \%$ PBS-T for $5 \mathrm{~min}$ and incubated in chromogenic substrate $[0.4 \mathrm{mg} / \mathrm{ml}$ AEC (3-aminoethyl-9-carbazole) in $0.5 \mathrm{M}$ acetate buffer with $0.1 \% \mathrm{H}_{2} \mathrm{O}_{2}, \mathrm{pH}$ 5.5]. After sufficient colouring the reaction was stopped by rinsing in water.

tPSA and fPSA determination. Total PSA (tPSA) and free PSA (fPSA) contents were determined by the immunochemistry analyser AIA 600 II (Tosoh, Japan). AIA 600 II is specifically designed for measurement of immunochemistry parameters in biological fluids using reagents of AIA-PACK series. Analyses were carried out according to the manufacturer's instructions. The instrument was calibrated using the AIA-PACK Calibrator set using a 6-point calibration (Tosoh). All reactions were performed in the special disposable test cups containing dried and lyophilized reagents. The immunochemical antigenantibody reaction employed magnetic particles $(1.5 \mathrm{~mm})$. Samples were incubated at $37^{\circ}$ C. 4-Methylumbelliferyl phosphate was used as a substrate and fluorescence corresponding to enzymatic activity on magnetic particles was measured.

Determination of serum caveolin-1 protein. For determination of the serum levels of caveolin-1 protein the Human caveolin-1 ELISA Kit (Uscn Life Science, Inc., Wuhan, China; detection range $0.24-15 \mathrm{ng} / \mathrm{ml}$ ) was used according to the manufacturer's manual. To detect the concentration of serum caveolin-1 level using the ELISA kit, the $60 \mathrm{ng} / \mathrm{ml}$ caveolin-1 standard was diluted to the concentration range $0.24-16 \mathrm{ng} / \mathrm{ml}$ in duplicates and absorption was measured.

Densitometric and statistical analysis. The signal intensity of bands after immunochemistry analysis was determined using the ImageJ 1.45 software (NIH, USA) as an area under the curve and concentration was calculated according to the protein 


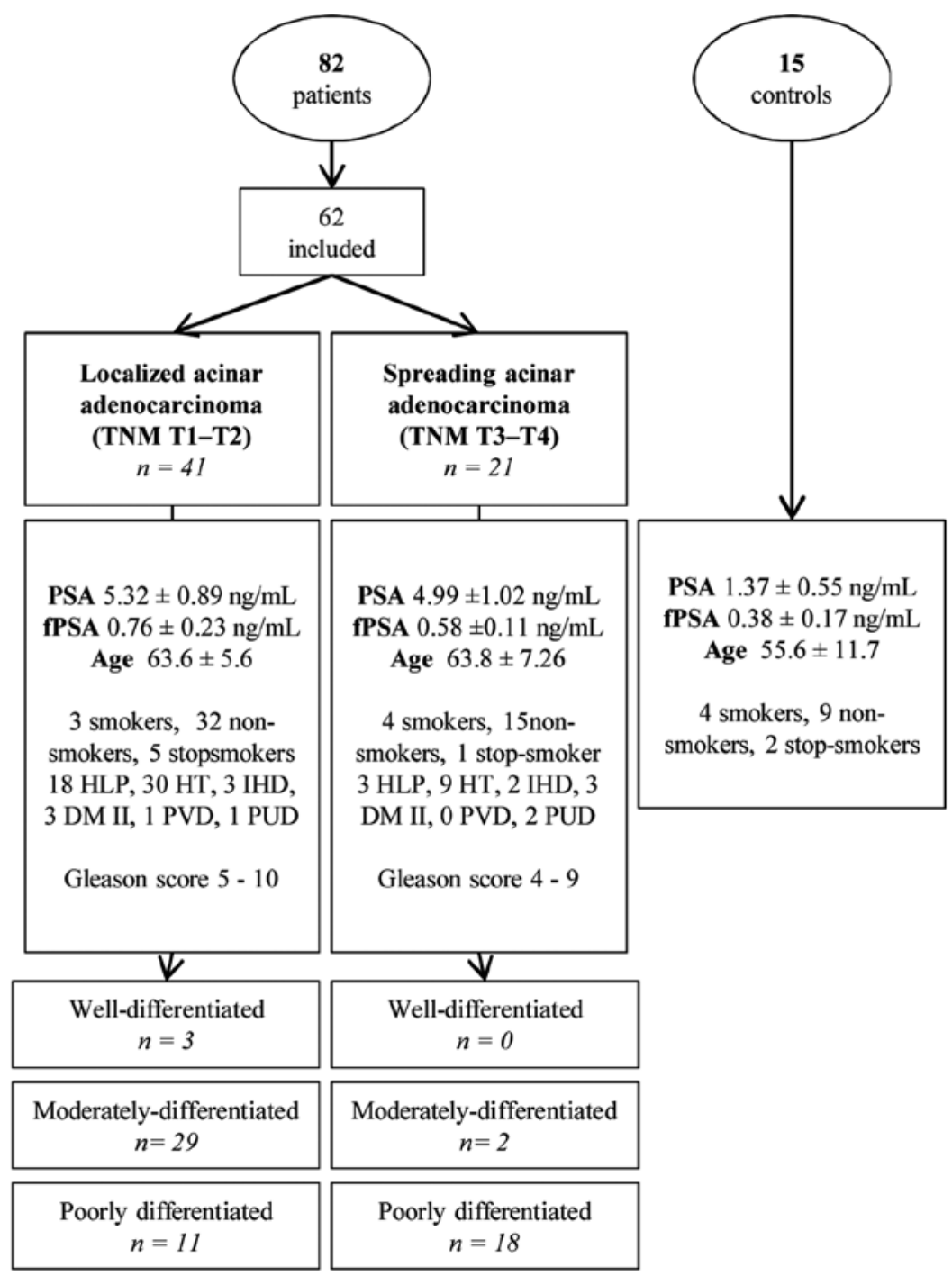

Figure 1. Characterisation of patients and controls. Categorization by tumour localization and differentiation. HLP, hyperlipoproteinaemia; HT, hypertension; IHD, ischaemic heart disease; DMII, diabetes mellitus type II; PVD, peripheral vascular diseas; PUD, peptic ulcer disease.

standard. Software Statistica 9.1 (StatSoft, USA) was used for statistical analysis. To test the normal distribution of data and thus usability of parametric tests, the Kolmogorov-Smirnov test was used. The Student's t-test for independent values was used to evaluate differences between the two groups. Simple linear correlations were performed to reveal the relationships between variables. To characterize data, associations were visualized using tree clustering with Euclidean distances measurement and single linkage. Subsequently, patients divided into clusters using K-means clustering analysis. Unless noted otherwise, a level of statistical significance was designated to $\mathrm{p}=0.05$.

\section{Results}

Blood serum caveolin-1 levels were statistically evaluated in groups of controls and histologically verified tumours. Consequently, caveolin-1 level was related to data in the patient's history, such as age, smoking habits, associated diseases, clinical tumour stage and histological grade (Fig. 1). Subsequently, caveolin-1 was related to the level of serum PSA in order to evaluate the use of caveolin-1 as an auxiliary marker along with a PSA. Cluster analysis of PSA and caveolin-1 levels was performed to divide the patients into the groups characterised with high or low caveolin-1 or PSA. In these groups tumour stage and grade was evaluated.

Because tumour pathogenesis and growth are tightly associated with oxidative stress, we also determined serum markers of antioxidant activity, GSH, 2,2-diphenyl-1-picrylhydrazyl (DPPH), Trolox ${ }^{\circledR}$ equivalent antioxidant capacity (TEAC), ferric reducing antioxidant power (FRAP), N,N-dimethyl-1,4diaminobenzene (DMPD), free radicals method (FRK) and blue chromium peroxide (Cro). We have previously described these methods in detail in study by Sochor et al (56). In addition, it has been shown that caveolin-1 is associated with oxidative stress in cancer progression. Thus, we correlated caveolin-1 levels with markers of oxidation and performed cluster analysis to elucidate possible associations. We also associated caveolin-1 with other previously determined potential prostate cancer serum tumour markers, namely $\alpha$-methylacyl-CoA racemase (AMACR) and metallothionein (MT) which we have previously demonstrated as a high specificity and sensitivity diagnostic tool for prostate cancer diagnosis (59). 
$\mathbf{A}$

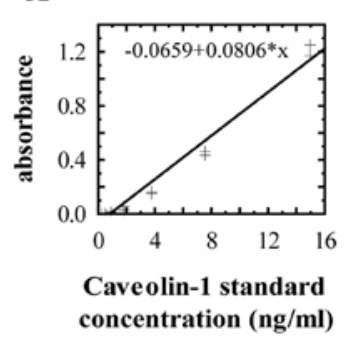

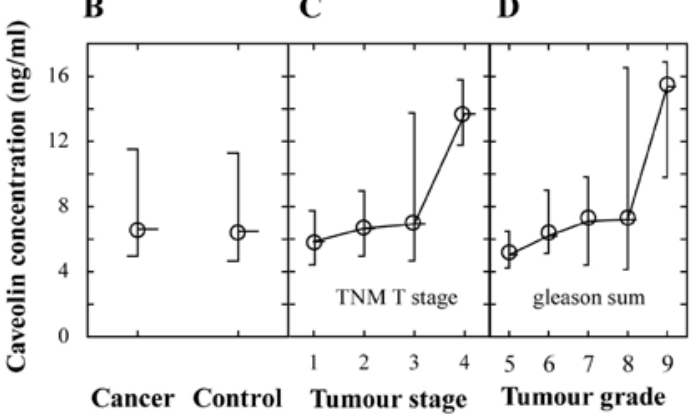

Figure 2. Caveolin-1 in patients. (A) Calibration curve. Absorbance of caveolin-1 standard in concentrations $0.24-16$ ng/ml, obtained calibration curve at correlation $r=0.98$. (B) Serum caveolin-1 in patients and controls. No significant difference between groups was observed. (C) Caveolin-1 in relation to TNM tumour stage. Significantly higher $(\mathrm{p}<0.004)$ caveolin-1 level was determined in tumour exceeding seminal vesicles $(\mathrm{T} 4)$ compared to lower stages of tumour. (D) Caveolin-1 grouped by histological tumour grade (Gleason sum). Higher caveolin-1 level was in high grade tumours (correlation $\mathrm{r}=0.29$ at $\mathrm{p}=0.028)$.

Table I. Caveolin-1 and PSA levels in relation to tumour stage (TNM T stage), histological differentiation and tumour grade (Gleason sum score).

n PSA $(\mathrm{ng} / \mathrm{ml}) \quad$ Free PSA $(\mathrm{ng} / \mathrm{ml}) \quad$ Caveolin-1 $(\mathrm{ng} / \mathrm{ml})$

\section{Tumour stage}

$\mathrm{T} 1$

$\mathrm{T} 2$

T3

T4

Tumour differentiation

Well
Moderately
Poorly

Tumour grade (Gleason sum score)

$$
5
$$

6

7

8

9

10

Total

$\begin{array}{rc}1 & 3.12 \\ 40 & 5.41 \pm 3.44 \\ 18 & 5.18 \pm 3.96 \\ 3 & 5.58 \pm 4.01\end{array}$

3

23

28

$\begin{array}{rc}5 & 3.69 \pm 1.43 \\ 20 & 5.03 \pm 2.56 \\ 32 & 4.85 \pm 3.22 \\ 3 & 10.12 \pm 8.17 \\ 4 & 6.87 \pm 5.03 \\ 1 & 3.90 \\ 62 & 4.83 \pm 3.56\end{array}$

$4.15 \pm 1.40$

$4.83 \pm 3.50$

$5.95 \pm 4.66$

$4.83 \pm 3.56$

\subsection{4}

$0.77 \pm 0.44$

$0.58 \pm 0.38$

$0.57 \pm 0.33$

$0.63 \pm 0.42$

$0.66 \pm 0.40$

$0.72 \pm 0.48$

$0.46 \pm 0.02$

$0.82 \pm 0.39$

$0.58 \pm 0.37$

$1.02 \pm 0.82$

$0.64 \pm 0.42$

$-\mathrm{b}$

$0.66 \pm 0.39$
$5.25 \pm 2.62$

$6.60 \pm 3.69$

$14.25 \pm 2.19^{\mathrm{a}}$

$4.13 \pm 1.27$

$5.64 \pm 3.02$

$6.28 \pm 3.32$

$3.86 \pm 1.11$

$5.11 \pm 2.42$

$5.75 \pm 2.98$

$6.96 \pm 4.83$

$9.99 \pm 4.04$

$-\mathrm{b}$

$5.65 \pm 3.02$

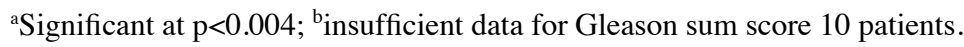

Tested cohort characteristics. We have analysed sera of 82 patients with histologically verified acinar adenocarcinoma of the prostate and 15 controls (Fig. 1). Out of these patients only 62 patients were selected for further analysis due to the lack of complete history data or insufficient quality of samples (haemolysis and/or extreme values detected). The average age of the patients was 63.7 years and ranged from 48 to 78 years. Of the 62 patients, 49 were non-smokers, 9 were smokers and the rest were former smokers. Forty-two patients $(68 \%)$ had localized tumours (T1-2) and 20 patients had spreading tumours of higher stage. The tumour differentiation ranged from well differentiated (3 tumours) to poorly differentiated (27 tumours) (Table I). The Gleason sum score ranged within 5-10. Patients had the following associated illnesses: hypertension (39 patients) hyperlipoproteinaemia (21 patients), ischaemic heart disease (5 patients), diabetes mellitus type II (6 patients), peripheral vascular disease (1 patient), peptic ulcer disease ( 3 patients), and tumour in history (1 patient). Of 62 patients, $15 \%$ did not suffer from any associated disease.

Caveolin-1 in patients and controls. Caveolin-1 standard in the concentration range from 0.24 to $16 \mathrm{ng} / \mathrm{ml}$ was used for determining the calibration dependence shown in Fig. 2A. Using linear regression we obtained strictly linear dependence with $\mathrm{R}^{2}>0.99$. The serum caveolin- 1 concentration ranged within $1.12-14.15 \mathrm{ng} / \mathrm{ml}$ and $1.74-14.97 \mathrm{ng} / \mathrm{ml}$ in the patients group and controls, respectively. The mean serum concentrations were $5.69 \mathrm{ng} / \mathrm{ml}$ in the cancer group and $5.42 \mathrm{ng} / \mathrm{ml}$ 

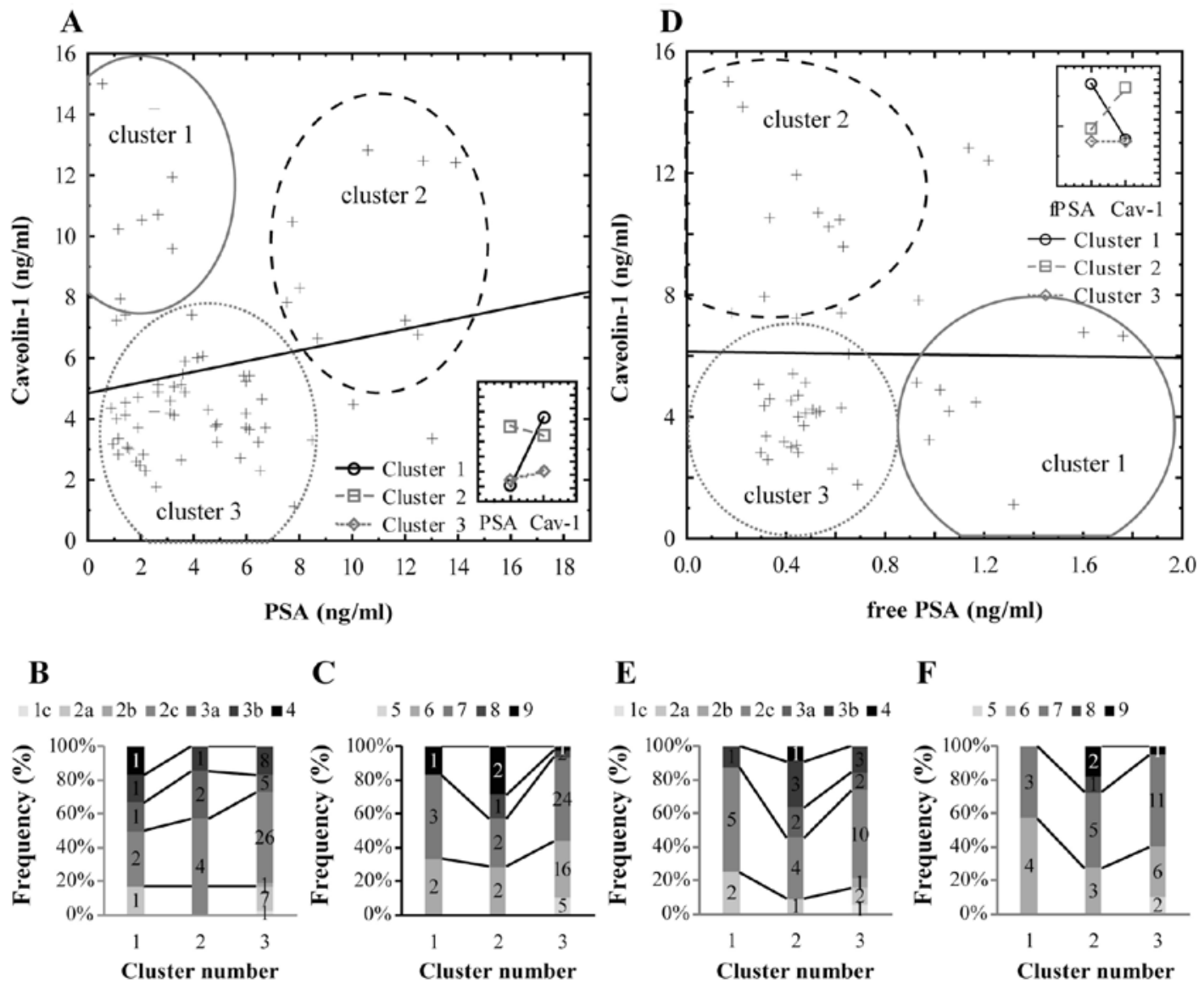

\section{C} $\because 5=6=7 \approx 8=9$

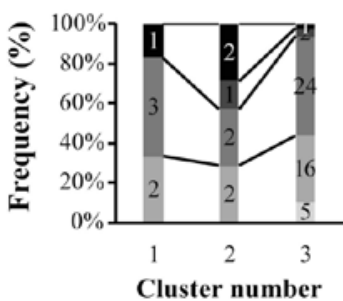

$\mathbf{E}$

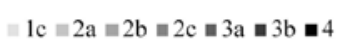

\section{F}

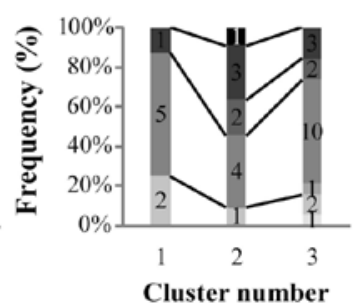

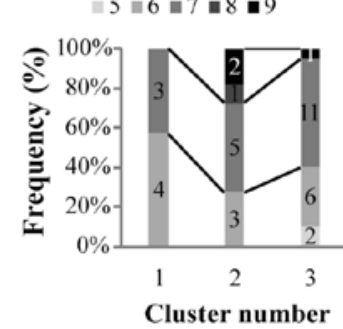

Figure 3. Caveolin-1 and prostate specific antigen (PSA). (A) Caveolin-1-PSA relation weak insignificant correlation was observed (see inset). Three clusters were apparent: patients with low PSA and high caveolin-1 (grey cluster 1), patients with high PSA and high caveolin-1 (dashed cluster 2) and patients with low PSA and low caveolin-1 (dotted grey cluster 3). (B) Tumour stage (TNM T) in three determined PSA/caveolin-1 clusters. Patients with low caveolin-1 and low PSA (cluster 3) had mostly lower grade tumours compared to clusters 1 and 2. (C) Tumour grade (Gleason sum) in PSA/caveolin-1 clusters of patients. Larger proportion of patients with higher PSA and higher caveolin-1 had higher grade (GS 8,9) tumours compared to patients with high caveolin-1 only (cluster 1) and low PSA and low caveolin-1 (cluster 3). (D) Caveolin-1 and free PSA no significant dependence revealed. Three clusters were apparent: patients with high free PSA and low caveolin-1 (grey cluster 1), patients with high caveolin-1 and low free PSA (dashed cluster 2) and patients with low caveolin-1 and low free PSA (dotted grey cluster 3). (E) Tumour stage (TNM T) in fPSA/caveolin-1 clusters. Patients with low fPSA and high caveolin-1 (cluster 2) had distinctly higher stage tumours compared to other clusters. (F) Tumour grade (Gleason sum) in fPSA/caveolin-1 clusters of patients. Patients with high caveolin-1 and low free PSA (cluster 2) had distinctly higher grade tumours.

in the control group. To verify the normality and thus suitability of parametric methods, the Kolmogorov-Smirnov test was performed. We found caveolin-1 levels were distributed normally $(\mathrm{p}<0.1)$, thus, independent $\mathrm{t}$-test was used. No significant change between group of controls and patients was determined (Fig. 2B). Cluster analysis revealed statistically significant variance of values in a group of cancer sera and led to classification of patients into two groups: high caveolin-1 and low caveolin-1 (data are not shown). Serum caveolin-1 was subsequently related to tumour stage (TNM T stage) and tumour grade (Gleason score sum) to clarify the differences in values. A similar trend was observed in both relations: caveolin-1 levels remained low in low stage and low grade tumours and distinctly increased in the highest stage and grade tumours (Fig. 2C and D). Patients with tumour spreading beyond the seminal vesicle (TNM T4 stage) had significantly $(\mathrm{p}<0.004)$ 2.8-fold increased serum caveolin-1 levels compared to T1-3 stages (Table I). However, no statistically significant differences between localised (T1-2) tumours and those that extend through the prostate capsule (T3-4) were detected. In terms of tumour grade and its relation to caveolin-1, patients with
Gleason sum 9 had distinctly higher caveolin-1 levels. This trend was significant $(\mathrm{r}=0.29$ at $\mathrm{p}=0.028)$; however, the difference between Gleason sum 9 patients and others was below the threshold of statistical significance.

In addition, other correlations with data obtained from history were carried out. No statistically important correlations between age of patients and caveolin-1 were found. Level of caveolin-1 was not significantly changed in connection with associated disease-hypertension, ischemic heart disease and hyperlipidaemia, ischemic disease of lower extremities and duodenal ulcer. Similarly, no differences in monitored markers between group of smokers and non-smokers were evident (data not shown).

Caveolin-1 and PSA correlation. Correlation analysis of serum caveolin-1 levels to PSA levels, found that no correlation exists between these proteins $(\mathrm{p}=0.13)$. When cluster analysis was performed, three groups were found: patients with low caveolin-1 and low PSA (grey dotted ellipse, cluster 3 in Fig. 3A), patients with low PSA and high caveolin-1 (grey cluster 1) and patients with high caveolin-1 and PSA (black dashed cluster 2). 


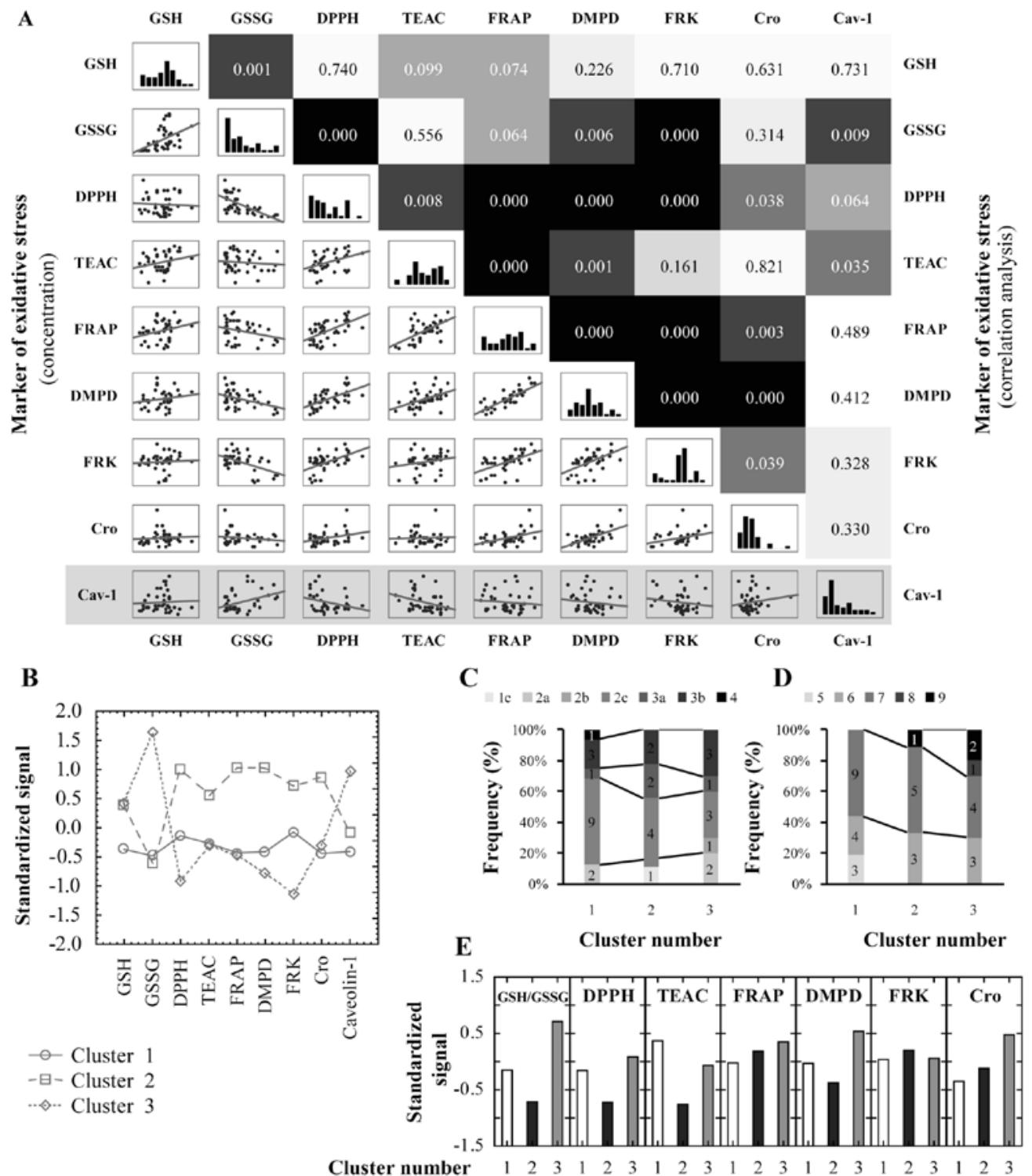

Figure 4. Caveolin-1 and markers of oxidation. (A) Correlations of markers of oxidation and caveolin-1 with each other with distribution histograms (diagonally). Bottom left part displays relations of markers/proteins, the top right part displays statistical significance of correlation (black, more significant trend; white, less significant). Evident significant correlations of caveolin-1 with reduced glutathione (GSH), 2,2-diphenyl-1-picrylhydrazyl (DPPH) and Trolox ${ }^{\circledR}$ equivalent antioxidant capacity (TEAC). (B) Cluster analysis of markers of oxidation together with caveolin-1. Inverse association of caveolin-1 and markers is obvious: the first cluster is characterized by no relations in caveolin-1 and markers of oxidation. The second cluster represents patients with high antioxidative potential (low GSSG and high antioxidative markers) and high caveolin-1. The third cluster represents patients with lower oxidative/antioxidant markers and low caveolin-1. (C) Tumour stage in subsequent clusters. No distinct differences in tumour staging within clusters of high or low caveolin-1/markers of oxidation were evident. (D) Tumour grade (represented as Gleason sum) in subsequent clusters. In the 'low antioxidative potential' cluster 3 are patients of distinctly higher content of high grade tumours compared to other patients. (E) Markers of antioxidant capacity in clusters by PSA/caveolin-1 characterized in Fig. 3A. We found significantly lower levels of the GSH/GSSG ratio, DPPH, TEAC and DMPD in the 'high PSA-high caveolin-1' cluster compared to the other clusters.

To characterize patients within those clusters, stage and grade was plotted in Fig. 3B and C. It is well evident that patients with high caveolin-1 and PSA (cluster 2) had lower proportions of localized tumours $\mathrm{T} 1$ and $\mathrm{T} 2$ compared to patients where only caveolin-1 is high and the PSA remains low (cluster 1), or where both proteins were low (cluster 3). A similar trend was evident in tumour grading (Fig. 3C), whereas in cluster 3 there was only a minimal proportion of high grade Gleason sum 8 and 9 tumours. In the 'both markers high cluster 2' the proportion of these high grades was about $40 \%$.

A similar association was also observed with caveolin-1 and the free PSA fraction (Fig. 3D). Similarly to the total PSA, in a group of low free PSA and low caveolin-1 (cluster 3) the proportion of low stage and low grade tumours was higher (Fig. 3E and F). Patients characterised with high serum free PSA and low caveolin-1 were of marginally higher stage and grade compared to the 'low free PSA-low caveolin-1' cluster 3 group, whereas, patients with high serum caveolin-1 and low free PSA were of significant proportion of T3-4 and grade Gleason sum 8 and 9 tumours.

Association of caveolin-1 and oxidative stress. Markers of antioxidant activity were determined in the sera of patients and correlated with serum caveolin-1 level to elucidate 


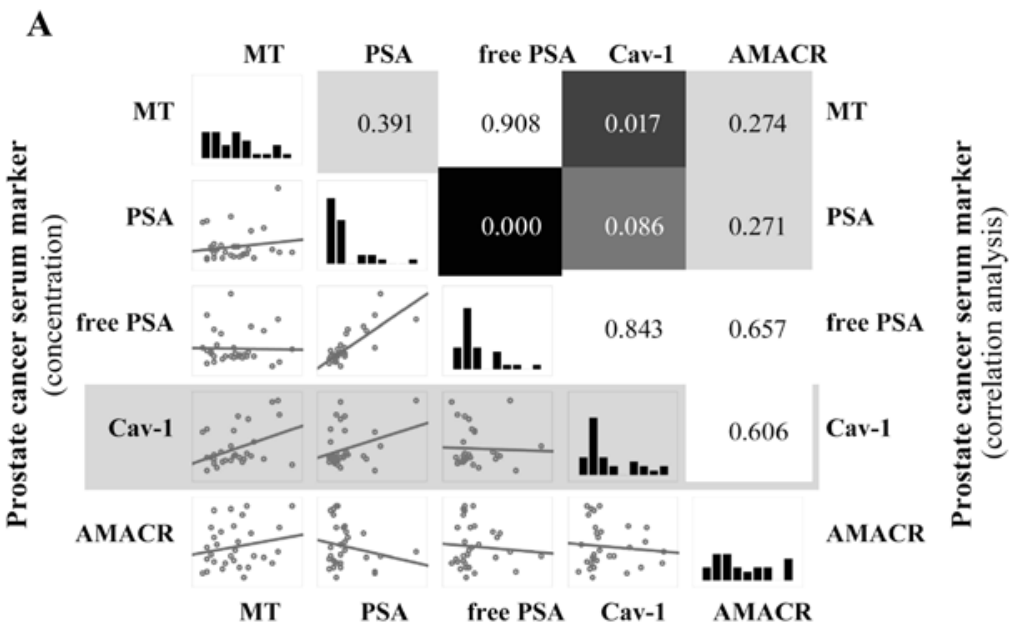

B

C
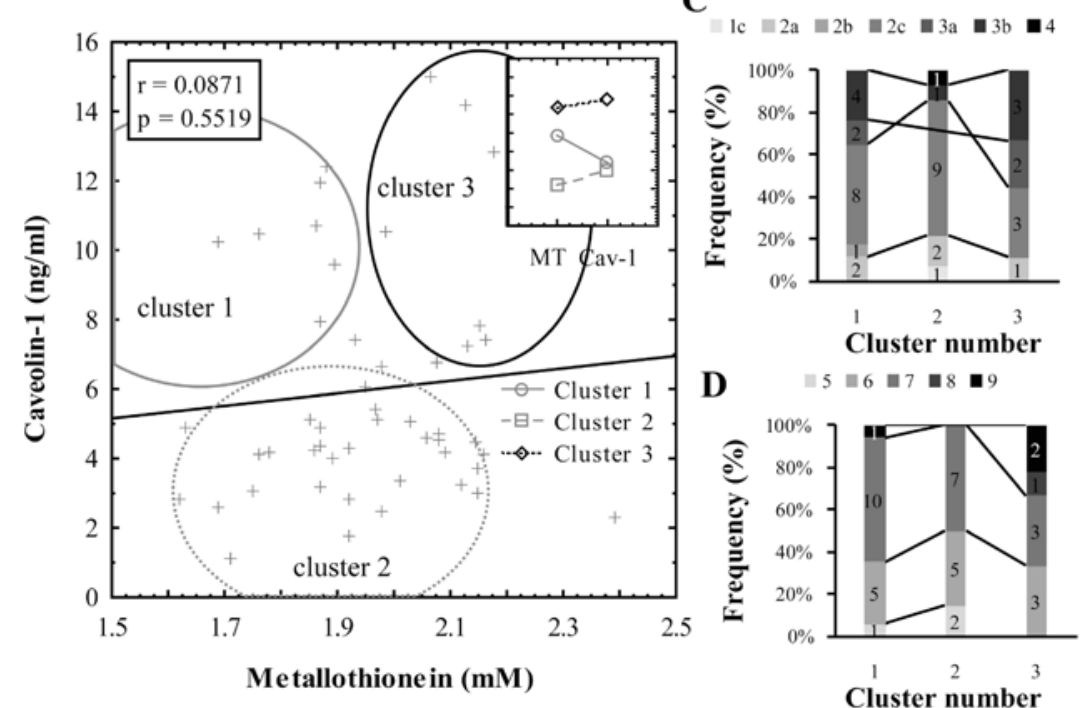

Figure 5. Relation of caveolin-1 to other potential prostate cancer markers. (A) Correlation matrix. A weak significant trend was observed between caveolin-1 and metallothionein (MT), no significant relation between caveolin-1 and PSA and $\alpha$-methylacyl CoA-racemase (AMACR). (B) Caveolin-1 and MT clustering analysis of clinical data. Three clusters were apparent: patients with higher MT and lower caveolin-1 (grey cluster 1), patients with low MT and low caveolin-1 (grey dotted cluster 2) and patients with high caveolin-1 and high MT (black cluster 3). (C) Tumour stage (TNM T) in MT/caveolin-1 clusters. Higher proportion of high-stage tumours are apparent in cluster 3 compared to cluster 2. (D) Tumour grade (Gleason sum) in clusters of patients. In the cluster with higher MT and higher caveolin-1 (cluster 3) a higher proportion of patients with high-grade tumours is apparent compared to the other clusters.

potential connections. Association between those markers is displayed in Fig. 4A. We observed significant trends between caveolin-1 and reduced GSH, TEAC and borderline significance with DPPH. Among others, our results show that most of the markers of antioxidant capacity correlate more with each other compared to with caveolin-1. Strongest correlations were observed between ferric reducing antioxidant power and $\mathrm{N}, \mathrm{N}$-dimethyl-1,4-diaminobenzene and free radical method. In order to elucidate the connections between markers of (anti)oxidation, cluster analysis was performed. Using this procedure, patients were divided into three distinct clusters (Fig. 4B) as follows. Cluster 2 which consists of patients with high antioxidant capacity, low caveolin-1 and low reduced GSH (Fig. 4B); cluster 3 which consists of patients with low antioxidative capacity, high caveolin-1 and high reduced glutathione and cluster 1 which shows no apparent dependencies compared to the previous clusters, and in which the markers of (anti)oxidation are of rather lower levels. In Fig. 4C and D we identified the tumour grade and tumour stage within these clusters. It is clear from these results that patients with lower antioxidative potential and higher caveolin-1 level (cluster 3) had a higher proportion of high grade (Gleason sum 8,9) tumours compared to others (compare clusters 2 and 3 in Fig. 4D). No similar trend was however observed, if we highlighted tumour stage within these clusters (Fig. 4C).

Then, we aimed our attention at the markers of antioxidative capacity from a different perspective. We related markers of antioxidation to caveolin-1 and PSA together. Three clusters of PSA have been described: high PSA and caveolin-1, high caveolin-1, and low PSA and caveolin-1. In these clusters, the levels of individual antioxidative markers were visualized. We found a significantly lower $(\mathrm{p}<0.05)$ level GSH/GSSG ratio, DPPH, TEAC and DMPD in the group of patients with high caveolin-1 and high PSA compared to the group of patients with low serum caveolin-1 and low PSA.

Association of caveolin-1 with other potential tumour markers. We subsequently performed correlation analyses 
between caveolin-1 and other potential serum markers of prostate cancer, which we have determined in our previous studies on the same sample set of patients (59). Thus, regarding PSA, which is mentioned in this table for overall complexity, here we show associations of caveolin-1 with MT and AMACR (Fig. 5A). In our previous study we have shown that metallothionein may be utilized as a marker of prostate cancer with high level of sensitivity and specifity (59). We found no major relationships between serum caveolin-1 level and serum MT levels ( $\mathrm{r}=0.08$, Fig. 5B). No trend was also observed when associated with AMACR. When cluster analysis on MT and caveolin-1 was carried out in the same way as with PSA or markers of oxidation (Fig. 5A), we found that when both of these serum markers are of high level, worse prognosis is expected, because greater proportion high grade (30 vs. $0 \%$ of GS 8-9 tumours in cluster 3 vs. cluster 2 ) and high stage (30 vs. $14 \%$ of T3b and T4 tumours in cluster 3 vs. cluster 2) tumours are present in these patients (Fig. 5C and D).

\section{Discussion}

In this study, we may clearly conclude that caveolin-1 is associated with worse prognosis. This we may evidence by three findings: first, higher serum caveolin-1 levels are associated with higher stage and grade tumours (which are of worse prognosis); second, caveolin-1 positively correlates with PSA levels, and third, patients with high serum caveolin-1 have a lower antioxidant capacity of the body. It is highly desirable to differentiate high-risk 'significant' forms of prostate cancer from latent, 'non-significant' forms. We expect that the prediction of high-risk tumours may be estimated when more cancer markers are determined concurrently. Utilization of caveolin-1 together with free and total PSA and possibly also with metallothionein may provide more accurate results in the estimation of prostate cancer risk when determined together. Based on our results we may conclude that patients with low free PSA, high caveolin-1 and high total PSA have worse prognosis compared to patients with lower serum caveolin-1 levels. Similarly, patients, who have high caveolin-1 and low PSA have better prognosis (lower stage and lower grade tumours) compared to patients with high PSA and caveolin-1 together. The similar benefit is also provided by the combined utilization of metallothionein and caveolin-1 as tumour markers, where patients with high metallothionein and low caveolin-1 are of better prognosis compared to patients with high levels of both markers. The merit of the use of fPSA, tPSA, caveolin-1 together as a marker of worse prognosis tumour may be underplayed by this finding. In cancers, the antioxidant capacity is reduced (51-53). Furthermore, patients with reduced antioxidant capacity are of worse prognosis when compared to patients with higher antioxidant potential. We may clearly confirm that the antioxidant capacity is associated with the severity of disease in this experiment by findings shown in Fig. 4D, from which it is apparent that patients with low antioxidative markers are of higher tumour grade.

An important finding of our study is the fact that high caveolin-1 levels are associated with patients of low antioxidative potential. Cluster analysis shown in Fig. 4B clearly points to the relationship between caveolin-1 and the antioxidative capacity of the patient's body. Patients with low caveolin-1 are of better prognosis because their antioxidative capacity is apparently higher (cluster 2 in Fig. 4B), whereas patients with high serum caveolin-1 have apparently lower antioxidative capacity. Due to the fact that caveolin-1 positively correlates with reduced glutathione and negatively correlates with markers of antioxidant capacity (even below the level of significance), we may speculate that caveolin-1 reflects the burden of the disease. This relation has not been published yet. The relationship of caveolin-1 with oxidative stress can also be viewed from another point of view. Recent studies have demonstrated that caveolin-1 is a target molecule in p38 MAPK mediated response to stress conditions such as oxidative stress $(27,28)$. After such stimuli, caveolin-1 is phosphorylated and thus contributes to various signalling pathways (60) resulting most likely in processes, such as premature cellular senescence (27). Thus, the connection of low antioxidant capacity and high caveolin-1 found in our study may be explained as consequence of caveolin-1 mediated response to high oxidative burden. In such long-term high oxidative stress conditions the resulting antioxidant capacity may be reduced, nevertheless, caveolin-1 remains higher. Utilization of multiple protocols for the determination of antioxidative capacity or free radical quenching activity, as performed in this study, may be useful because of discrepancies between the activity measured in vitro, and the antioxidative effects observed in vivo (61). Each technique is based on different principles and enables determination of the antioxidant activity of specific groups of compounds $(56,62,63)$.

In the recent decade, caveolin-1 was linked with various types of cancers, of which it was most extensively studied in the cancers of the breast and prostate. It was found that tumour tissue and/or serum caveolin-1 levels vary in a cancer-dependent manner and these changes in caveolin-1 levels may be associated with tumour protection or progression (25). When focused on prostate cancer, elevated caveolin-1 expression was observed in tumour tissue and in mice when compared to non-tumour tissue (64-66). According to Thompson et al, caveolin-1 is also elevated in the serum of patients with localized tumours compared to healthy controls and patients with benign prostatic hyperplasia $(67,68)$. These findings are inconsistent with ours; we found no significant difference between controls and cancers. However, it has also been revealed that caveolin-1 is connected with tumour progression and metastatic dissemination and is distinctly elevated in androgen resistant tumours. Thus this molecule was suggested as a tissue marker of an aggressive form of cancer (68-74). It has also been shown that suppression of caveolin-1 expression can restore the sensitivity to androgens in androgen-insensitive tumours (65). Higher expression of caveolin-1 was also identified in tissue samples of patients of higher grade tumours and of higher PSA (75). This is in good agreement with our results, where we also identified associations with high stage and high grade tumours with high PSA. Also, according to a study by Karam et al on patients before and after radical prostatectomy, patients of higher caveolin-1 are in risk of higher postoperative PSA and thus of worse postoperative prognosis (75). Although we did not compare patients before and after treatment procedures, we similarly observed correlation of caveolin-1 with PSA. Furthermore, worse prognosis could be expected from higher oxidative stress in high caveolin-1 patients. 
Our findings together with data from recent studies suggest that caveolin-1 is involved in disease pathogenesis and progression. Clarification can contribute to the understanding of this disease with potential novel targeted therapeutic approaches. Although associations between caveolin-1 and high-risk tumours were identified in this study, we still cannot infer that caveolin-1 may serve as a high-risk aggressive tumour marker even in a phase when tumours are localized in the prostate and thus are still curable. To confirm this, it is necessary to monitor caveolin-1 levels in a follow-up study during the course of prostate cancer progression from its initial stages. It is necessary to verify these facts in the extensive group of patients including those with disseminated disease.

\section{Acknowledgements}

Financial support from IGA MZ NS10200-3 and CEITEC CZ.1.05/1.1.00/02.0068 is greatly acknowledged.

\section{References}

1. Gannot G, Tangrea MA, Richardson AM, et al: Layered expression scanning: multiplex molecular analysis of diverse life science platforms. Clin Chim Acta 376: 9-16, 2007.

2. Bensalah K, Montorsi F and Shariat SF: Challenges of cancer biomarker profiling. Eur Urol 52: 1601-1609, 2007.

3. Duffy MJ: PSA as a marker for prostate cancer: a critical review. Ann Clin Biochem 33: 511-519, 1996.

4. Matsumoto K, Irie A, Satoh T, Kuruma H, Arakawa T and Baba S: Occupational bladder cancer: from cohort study to biologic molecular marker. Med Sci Monitor 11: RA311-RA315, 2005.

5. Sargent DJ, Conley BA, Allegro C and Collette L: Clinical trial designs for predictive marker validation in cancer treatment trials. J Clin Oncol 23: 2020-2027, 2005.

6. Sarrazin S, Adam E, Lyon M, et al: Endocan or endothelial cell specific molecule-1 (ESM-1): a potential novel endothelial cell marker and a new target for cancer therapy. Biochim Biophys Acta 1765: 25-37, 2006.

7. Tuxen MK: Tumor marker CA125 in ovarian cancer. J Tumor Marker Oncol 16: 49-68, 2001.

8. Zehentner BK and Carter D: Mammaglobin: a candidate diagnostic marker for breast cancer. Clin Biochem 37: 249-257, 2004

9. Barry MJ: Prostate-specificy-antigen testing for early diagnosis of prostate cancer. N Engl J Med 344: 1373-1377, 2001.

10. Catalona WJ, Smith DS, Ratliff TL, et al: Measurement of prostate-specific antigen in serum as a screening test for prostate cancer. N Engl J Med 324: 1156-1161, 1991.

11. Thompson IM, Pauler DK, Goodman PJ, et al: Prevalence of prostate cancer among men with a prostate-specific antigen level $\leq 4.0 \mathrm{ng}$ per milliliter. N Engl J Med 350: 2239-2246, 2004.

12. Schmid HP, McNeal JE and Stamey TA: Observations on the doubling time of prostate-cancer: the use of serial prostatespecific antigen in patients with untreated disease as a measure of increasing cancer volume. Cancer 71: 2031-2040, 1993.

13. Palade GE: Fine structure of blood capillaries. J Appl Phys 24: $1424-1424,1953$

14. Patra SK and Bettuzzi S: Epigenetic DNA-methylation regulation of genes coding for lipid raft-associated components: A role for raft proteins in cell transformation and cancer progression (Review). Oncol Rep 17: 1279-1290, 2007.

15. Gould ML, Williams G and Nicholson HD: Changes in caveolae, caveolin, and polymerase 1 and transcript release factor (PTRF) expression in prostate cancer progression. Prostate 70: 1609-1621, 2010.

16. Couet J, Belanger MM, Roussel E and Drolet MC: Cell biology of caveolae and caveolin. Adv Drug Deliv Rev 49: 223-235, 2001.

17. Frank PG, Pavlides S and Lisanti MP: Caveolae and transcytosis in endothelial cells: role in atherosclerosis. Cell Tissue Res 335: 41-47, 2009.

18. Zheng YZ and Foster LJ: Biochemical and proteomic approaches for the study of membrane microdomains. J Proteomics 72: 12-22, 2009.
19. Volonte D, Galbiati F and Lisanti MP: Visualization of caveolin-1, a caveolar marker protein, in living cells using green fluorescent protein (GFP) chimeras. The subcellular distribution of caveolin-1 is modulated by cell-cell contact. FEBS Lett 445: 431-439, 1999.

20. UniProt knowledgebase for Q03135 (Cav1_Human). http://www. uniprot.org/uniprot/Q03135.

21. Shatz M and Liscovitch M: Caveolin-1: a tumor-promoting role in human cancer. Int J Radiat Biol 84: 177-189, 2008.

22. Burgermeister E, Liscovitch M, Rocken C, Schmid RM and Ebert MPA: Caveats of caveolin-1 in cancer progression. Cancer Lett 268: 187-201, 2008.

23. Ando T, Ishiguro H, Kimura M, et al: The overexpression of caveolin-1 and caveolin-2 correlates with a poor prognosis and tumor progression in esophageal squamous cell carcinoma. Oncol Rep 18: 601-609, 2007.

24. Zhang Q, Furukawa K, Chew HH, et al: Down-regulation of caveolin-1 in mouse Lewis lung cancer P29 is a causal factor for the malignant properties in a high-metastatic subline. Oncol Rep 16: 289-294, 2006

25. Williams TM and Lisanti MP: Caveolin-1 in oncogenic transformation, cancer, and metastasis. Am J Physiol Cell Physiol 288: C494-C506, 2005.

26. Conde MC, Ramirez-Lorca R, Lopez-Jamar JME, et al: Genetic analysis of caveolin-1 and eNOS genes in colorectal cancer. Oncol Rep 16: 353-359, 2006.

27. Dasari A, Bartholomew JN, Volonte D and Galbiati F: Oxidative stress induces premature senescence by stimulating caveolin-1 gene transcription through $\mathrm{p} 38$ mitogen-activated protein kinase/ Sp1-mediated activation of two GC-rich promoter elements. Cancer Res 66: 10805-10814, 2006.

28. Chen DB, Li SM, Qian XX, Moon CS and Zheng J: Tyrosine phosphorylation of caveolin 1 by oxidative stress is reversible and dependent on the c-src tyrosine kinase but not mitogen-activated protein kinase pathways in placental artery endothelial cells. Biol Reprod 73: 761-772, 2005.

29. Krizkova S, Adam V, Petrlova J, et al: A suggestion of electrochemical biosensor for study of platinum(II)-DNA interactions. Electroanalysis 19: 331-338, 2007.

30. Popiela TJ, Rudnicka-Sosin L, Dutsch-Wicherek M, et al: The metallothionein and RCAS1 expression analysis in breast cancer and adjacent tissue regarding the immune cells presence and their activity. Neuro Endocrinol Lett 27: 786-794, 2006.

31. Zelena J, Potesil D, Vacek J, et al: Metallothionein as a prognostic marker of tumor disease. Klin Onkol 17: 190-195, 2004.

32. Ebert MPA, Günther T, Hoffmann J, et al: Expression of metallothionein II in intestinal metaplasia, dysplasia, and gastric cancer. Cancer Res 60: 1995-2001, 2000.

33. Kasahara K, Fujiwara Y, Nishio K, et al: Metallothionein content correlates with the sensitivity of human small cell lung cancer lines to cisplatin. Cancer Res 51: 3237-3242, 1991.

34. Theocharis SE, Margeli AP, Klijanienko JT and Kouraklis GP: Metallothionein expression in human neoplasia. Histopathology 45: 103-118, 2004.

35. Adam V, Baloun J, Fabrik I, Trnkova L and Kizek R: An electrochemical detection of metallothioneins at the zeptomole level in nanolitre volumes. Sensors 8: 2293-2305, 2008

36. Adam V, Blastik O, Krizkova S, et al: Application of the Brdicka reaction in determination of metallothionein in patients with tumours. Chem Listy 102: 51-58, 2008.

37. Fabrik I, Krizkova S, Huska D, et al: Employment of electrochemical techniques for metallothionein determination in tumour cell lines and patients with a tumor disease. Electroanalysis 20: 1521-1532, 2008.

38. Eckschlager T, Adam V, Hrabeta J, Figova K and Kizek R: Metallothioneins and cancer. Curr Protein Pept Sci 10: 360-375, 2009.

39. Adam V, Fabrik I, Eckschlager T, Stiborova M, Trnkova L and Kizek R: Vertebrate metallothioneins as target molecules for analytical techniques. TrAC-Trends Anal Chem 29: 409-418, 2010.

40. Adam V, Petrlova J, Wang J, Eckschlager T, Trnkova L and Kizek R: Zeptomole electrochemical detection of metallothioneins. PLoS One 5: e11441, 2010.

41. Ryvolova M, Krizkova S, Adam V, et al: Analytical methods for metallothionein detection. Curr Anal Chem 7: 243-261, 2011.

42. Kizek R, Trnkova L and Palecek E: Determination of metallothionein at the femtomole level by constant current stripping chronopotentiometry. Anal Chem 73: 4801-4807, 2001. 
43. Cherian MG, Jayasurya A and Bay BH: Metallothioneins in human tumors and potential roles in carcinogenesis. Mutat Res 533: 201-209, 2003.

44. Jin R, Huang J, Tan PH and Bay BH: Clinicopathological significance of metallothioneins in breast cancer. Pathol Oncol Res 10 74-79, 2004.

45. Kelly SL, Basu A, Teicher BA, Hacker MP, Hamer DH and Lazo JS: Overexpression of metallothionein confers resistance to anticancer drugs. Science 241: 1813-1815, 1988.

46. Theocharis S, Karkantaris C, Philipides T, et al: Expression of metallothionein in lung carcinoma: correlation with histological type and grade. Histopathology 40: 143-151, 2002.

47. Kuefer R, Varambally S, Zhou M, et al: $\alpha$-methylacyl-CoA racemase: expression levels of this novel cancer biomarker depend on tumor differentiation. Am J Pathol 161: 841-848, 2002.

48. Luo J, Zha S, Gage WR, et al: $\alpha$-methylacyl-CoA racemase: a new molecular marker for prostate cancer. Cancer Res 62: 2220$2226,2002$.

49. Xu J, Stolk JA, Zhang X, et al: Identification of differentially expressed genes in human prostate cancer using subtraction and microarray. Cancer Res 60: 1677-1682, 2000.

50. Evans AJ: $\alpha$-methylacyl CoA racemase (P504S): overview and potential uses in diagnostic pathology as applied to prostate needle biopsies. J Clin Pathol 56: 892, 2003.

51. Buttke TM and Sandstrom PA: Oxidative stress as a mediator of apoptosis. Immunol Today 15: 7-10, 1994.

52. Droge W: Free radicals in the physiological control of cell function. Physiol Rev 82: 47-95, 2002

53. Valko M, Rhodes CJ, Moncol J, Izakovic M and Mazur M: Free radicals, metals and antioxidants in oxidative stress-induced cancer. Chem Biol Interact 160: 1-40, 2006.

54. Potesil D, Petrlova J, Adam V, et al: Simultaneous femtomole determination of cysteine, reduced and oxidized glutathione, and phytochelatin in maize (Zea mays L.) kernels using high-performance liquid chromatography with electrochemical detection. J Chromatogr A 1084: 134-144, 2005.

55. Petrlova J, Mikelova R, Stejskal K, et al: Simultaneous determination of eight biologically active thiol compounds using gradient elution-liquid chromatography with Coul-Array detection. J Sep Sci 29: 1166-1173, 2006.

56. Sochor J, Ryvolova M, Krystofova O, et al: Fully automated spectrometric protocols for determination of antioxidant activity: advantages and disadvantages. Molecules 15: 8618-8640, 2010.

57. Laemmli UK: Cleavage of structural proteins during assembly of head of bacteriophage-T4. Nature 227: 680-685, 1970.

58. Merril CR, Dunau ML and Goldman D: A rapid sensitive silver stain for polypeptides in polyacrylamide gels. Anal Biochem 110: 201-207, 1981.

59. Krizkova S, Ryvolova M, Gumulec J, et al: Electrophoretic fingerprint metallothionein analysis as a potential prostate cancer biomarker. Electrophoresis 32: 1952-1961, 2011.

60. Volonte D, Galbiati F, Pestell RG and Lisanti MP: Cellular stress induces the tyrosine phosphorylation of caveolin-1 $(\operatorname{Tyr}(14))$ via activation of p38 mitogen-activated protein kinase and c-Src kinase - Evidence for caveolae, the actin cytoskeleton, and focal adhesions as mechanical sensors of osmotic stress. J Biol Chem 276: 8094-8103, 2001
61. Duplancic D, Kukoc-Modun L, Modun D and Radic N: Simple and rapid method for the determination of uric acid-independent antioxidant capacity. Molecules 16: 7058-7067, 2011.

62. Bartosz G: Non-enzymatic antioxidant capacity assays: limitations of use in biomedicine. Free Radic Res 44: 711-720, 2010.

63. Prior RL and Cao GH: In vivo total antioxidant capacity: comparison of different analytical methods. Free Radic Biol Med 27: 1173-1181, 1999.

64. Tahir SA, Ren CZ, Timme TL, et al: Development of an immunoassay for serum caveolin-1: a novel biomarker for prostate cancer. Clin Cancer Res 9: 3653-3659, 2003.

65. Nasu Y, Timme TL, Yang G, et al: Suppression of caveolin expression induces androgen sensitivity in metastatic androgeninsensitive mouse prostate cancer cells. Nat Med 4: 1062-1064, 1998.

66. Yang G, Truong LD, Wheeler TM and Thompson TC: Caveolin-1 expression in clinically confined human prostate cancer: a novel prognostic marker. Cancer Res 59: 5719-5723, 1999.

67. Tahir SA, Frolov A, Hayes TG, et al: Preoperative serum caveolin-1 as a prognostic marker for recurrence in a radical prostatectomy cohort. Clin Cancer Res 12: 4872-4875, 2006.

68. Thompson TC, Tahir SA, Li L, et al: The role of caveolin-1 in prostate cancer: clinical implications. Prostate Cancer Prostatic Dis 13: 6-11, 2010.

69. Watanabe M, Yang G, Cao GW, et al: Functional analysis of secreted caveolin-1 in mouse models of prostate cancer progression. Mol Cancer Res 7: 1446-1455, 2009.

70. Tahir SA, Yang G, Ebara S, et al: Secreted caveolin-1 stimulates cell survival/clonal growth and contributes to metastasis in androgen-insensitive prostate cancer. Cancer Res 61: 3882-3885, 2001.

71. Yang G, Truong LD, Timme TL, et al: Elevated expression of caveolin is associated with prostate and breast cancer. Clin Cancer Res 4: 1873-1880, 1998

72. Thompson TC, Timme TL, Li L and Goltsov A: Caveolin-1, a metastasis-related gene that promotes cell survival in prostate cancer. Apoptosis 4: 233-237, 1999.

73. Li LK, Yang G, Ebara S, et al: Caveolin-1 mediates testosteronestimulated survival/clonal growth and promotes metastatic activities in prostate cancer cells. Cancer Res 61: 4386-4392, 2001.

74. Mouraviev V, Li LK, Tahir SA, et al: The role of caveolin-1 in androgen insensitive prostate cancer. J Urol 168: 1589-1596, 2002.

75. Karam JA, Lotan Y, Roehrborn CG, Ashfaq R, Karakiewicz PI and Shariat SF: Caveolin-1 overexpression is associated with aggressive prostate cancer recurrence. Prostate 67: 614-622, 2007. 